\title{
Correlation Functions of Quantum Integrable Models
}

\author{
J. M. Maillet \\ Groupe de Physique Théorique, \\ Laboratoire de Physique*. ENS Lyon, 46 Allée d'Italie, 69364 Lyon, France. \\ E-mail: 'maillet@ens-lyon.fir
}

ABSTRACT: A general approach to the computation of exact correlation functions of lattice quantum integrable models proposed recently is reviewed. It is based on the resolution of the so-called quantum inverse scattering problem, namely, the reconstruction of local operators in terms of the (operator) entries of the quantum monodromy matrix obeying a Yang-Baxter algebra. The application of this method to the $X X Z$ Heisenberg spin- $\frac{1}{2}$ chain in a magnetic field leads to multiple integral representations of its $n$-point correlation functions. For zero magnetic field, it gives a proof, in both the massless and massive (anti-ferromagnetic) regimes, of the formulas obtained from the q-deformed $\mathrm{KZ}$ equations (massless regime) and the representation theory of the quantum affine algebra $\mathcal{U}_{q}\left(\hat{s} l_{2}\right)$ together with the corner transfer matrix approach (massive regime).

\section{Introduction}

In the last twenty years considerable progress have been achieved in our understanding of classical and quantum integrable models of field theory and statistical mechanics [1-6]. New concepts and methods have emerged from this domain, leading to major breakthroughs not only in theoretical physics but also in mathematics. In this context, one of the main challenge of the theory of quantum integrable models remained largely open until now despite the great amount of remarkable works which have been devoted to its resolution: computing exact and manageable expressions for their correlation functions. This is a fundamental problem, both to enlarge the range of applications of these models, for example in the realm of condensed matter physics, and also to understand in a deeper way their underlying mathematical structures.

For a long time, only very few models were known for which correlation function can be computed in an exact and explicit way. The typical examples are the Ising model [14-16], re-

*UMR 5672 du CNRS associée à l'ENS Lyon. This work is supported by the european contract EC-TMRFMRX-CT96-0012 lated to free fermions, and conformal field theories dealing with critical or massless systems in the continuum $[17,18]$. Besides these models (for which series of formidable works have been necessary to obtain exact correlation functions), in the class of integrable models solvable by means of Bethe ansatz, and in particular for integrable lattice models, two different but complementary approaches have been considered to solve this problem.

One of them relies on the study of infinite dynamical symmetries of integrable models directly in the infinite volume limit. It started with the solution of bootstrap equations for $S$ matrices and form factors of (massive) relativistic quantum field theories [4,19-21] and merged with representation theory of the associated Yangians or quantum affine symmetries [22-28]. Using the additionnal ideas coming from two-dimensionnal statistical models resolution (like the Baxter corner transfer matrix method) [1,29-31], it leads (using however some hypothesis) to multiple integral representations of correlation functions of the $X X Z$ Heisenberg spins chains (at zero temperature and zero magnetic field) in the massive regime $[6,32,33]$. These functions being there re- 
lated to trace of products of $q$-deformed vertex operators satisfying $q$-deformed KZ-equations, it also led to conjectures for other regimes (gapless region) for which the corner transfer matrix approach is not available. More general models have been considered since then using this method.

Another approach, described essentially in the book [5], is based on the algebraic Bethe ansatz analysis [1,2,5,12] of these lattice models in the finite volume. However due to the complicated combinatorial structure of Bethe eigenstates and of the scalar products of general states, it did not succeed in obtaining explicit and manageable expressions for the correlation functions for generic models. Instead, this method seems to give insights into the long distance asymptotics of correlation functions [34].

The aim of this lecture is to review an approach proposed recently in [7-9] (see also [10, 11] for associated works) to compute correlation functions of lattice quantum integrable models using the algebraic Bethe ansatz method [1,2, $5,12]$ and the explicit resolution of the quantum inverse scattering problem [7,13], namely, the reconstruction of the local quantum operators (the correlation functions of which we want to calculate) in terms of the elements of the monodromy matrix of the model satisfying a YangBaxter algebra and containing in particular creation/annihilation operators of the Bethe eigenstates. Although this method has been developed first in the example of the Heisenberg spin$\frac{1}{2} X X Z$ chain [7], it is clear already that the basic tools and techniques used there can be applied in principle to generic quantum integrable lattice models (see [13] for a solution of the quantum inverse scattering problem for a large class of integrable lattice models).

\section{General approach to correlation functions}

Correlation functions of a certain product of local operators denoted generically by $\mathcal{O}$ is defined by,

$$
\frac{\operatorname{tr}_{\mathcal{H}}\left(\mathcal{O} e^{-\mathbf{H} / k T}\right)}{\operatorname{tr}_{\mathcal{H}}\left(e^{-\mathbf{H} / k T}\right)}
$$

where $T$ is the temperature, $k$ the Boltzmann constant and $H$ is the Hamiltonian of the model acting in the quantum space $\mathcal{H}$. In the zero temperature limit, only the groundstate $\left|\psi_{g}\right\rangle$ of $H$ contributes to the above trace, and the correlation function reduces to,

$$
\frac{\left\langle\psi_{g}|\mathcal{O}| \psi_{g}\right\rangle}{\left\langle\psi_{g} \mid \psi_{g}\right\rangle}
$$

Let us first review the two main difficulties in trying to compute such a quantity. The first problem to solve is of course the determination of the ground state $\left|\psi_{g}\right\rangle$. For interacting theories, this is already an highly non-trivial (and in general non-perturbative) problem. For integrable models solvable by means of algebraic Bethe ansatz $[1,2,5,12]$, it admits a solution in terms of creation (resp. annihilation) operators which are elements of the associated quantum monodromy matrix $T_{i}(\lambda) \in \operatorname{End}\left(V_{i}\right) \otimes \mathcal{H}$ satisfying the Yang-Baxter algebra $[35,36]$,

$$
\begin{aligned}
& R_{12}(\lambda, \mu) T_{1}(\lambda) T_{2}(\mu)= \\
& =T_{2}(\mu) T_{1}(\lambda) R_{12}(\lambda, \mu),
\end{aligned}
$$

with the usual tensor notations $T_{1}(\lambda)=T(\lambda) \otimes \operatorname{Id}$ and $T_{2}(\mu)=\operatorname{Id} \otimes T(\mu), R$ being a linear operator in the tensor product of two two-dimensional linear spaces $V_{1} \otimes V_{2}$ and obeying the Yang-Baxter equation,

$$
\begin{aligned}
& R_{12}\left(\lambda_{1}, \lambda_{2}\right) R_{13}\left(\lambda_{1}, \lambda_{3}\right) R_{23}\left(\lambda_{2}, \lambda_{3}\right)= \\
= & R_{23}\left(\lambda_{2}, \lambda_{3}\right) R_{13}\left(\lambda_{1}, \lambda_{3}\right) R_{12}\left(\lambda_{1}, \lambda_{2}\right),
\end{aligned}
$$

In the most simple cases the monodromy matrix is a $2 \times 2$ matrix in the auxiliary space $V$, with operator valued entries acting in $\mathcal{H}$,

$$
T(\lambda)=\left(\begin{array}{ll}
A(\lambda) & B(\lambda) \\
C(\lambda) & D(\lambda)
\end{array}\right)
$$

The Hamiltonian is then obtained as a member of the commuting set of operators generated by the transfer matrix $A(\lambda)+D(\lambda)$, namely the trace of the above monodromy matrix, which are commuting operators for arbitrary values of the spectral parameter $\lambda$. The ground state is then constructed by the multiple action of the $B$ operators on a known reference state $|0\rangle$ as for example, $B\left(\lambda_{1}\right) \ldots B\left(\lambda_{N}\right)|0\rangle$ (and for the dual state, 
$\left.\langle 0| C\left(\lambda_{1}\right) \ldots C\left(\lambda_{N}\right)\right)$ which is a common eigenstate of the transfer matrices if the set of spectral parameters $\left\{\lambda_{j}\right\}_{1 \leqslant j \leqslant N}$ is a solution of the Bethe equations [37],

$$
\frac{a\left(\lambda_{j}\right)}{d\left(\lambda_{j}\right)} \prod_{\substack{k=1 \\ k \neq j}}^{N} \frac{b\left(\lambda_{j}, \lambda_{k}\right)}{b\left(\lambda_{k}, \lambda_{j}\right)}=1, \quad 1 \leqslant j \leqslant N
$$

where $a(\lambda)$ and $d(\lambda)$ are the eigenvalues of operators $A(\lambda)$ and $D(\lambda)$ respectively on the reference state $|0\rangle$ and $b(\lambda, \mu)$ is a function of two parameters given by the $R$-matrix.

The second problem is to express in a compact way the action of any product of local operator $\mathcal{O}$ on such Bethe eigenstates. This question remained open for many years. The reason is the following. Although the operators $B(\lambda)$ satisfy together with the other elements of the monodromy matrix a nice quadratic algebra (2.3i), they are highly non-local quantities in terms of the local operators defining the model. Hence, there was until recently no way to obtain the needed algebra between local operators and the monodromy matrix operator entries. It turns out that for almost all known lattice quantum integrable models, this problem has a simple and explicit solution: it is to reconstruct local operators in terms of the monodromy matrix entries, or in other words to solve explicitly the so-called quantum inverse scattering problem. We will give in the following the formulas corresponding to the particular case of the $X X Z$ spin- $\frac{1}{2}$ Heisenberg finite chain [7]. However, the simplicity of the result and of its proof opened the way for generalisation to almost all known lattice integrable models [13]. This reconstruction of the local operators will give the key to the computation of the correlation functions.

To achieve this goal, our method will be decomposed into the following main steps:

1 - Construction of the groundstate $\left|\psi_{g}\right\rangle$ in the algebraic Bethe ansatz framework as, $\left\langle\psi_{g}\right|=$ $\langle 0| \prod_{i \in I} C\left(\lambda_{i}\right)$ and $\left|\psi_{g}\right\rangle=\prod_{i \in I} B\left(\lambda_{i}\right)|0\rangle,|0\rangle$ being some reference state, and the parameters $\lambda_{i}, i \in$ $I$, satisfying Bethe equations.
2 - Algebraic solution of the quantum inverse scattering problem for local operators, namely their reconstruction in terms of the monodromy matrix operator entries.

3 - Expression of the action on the ground state of any product of local operators using their expression in terms of the monodromy matrix and the Yang-Baxter algebra it satisfies, as multiple sums,

$\left\langle\psi_{g}\right| \mathcal{O}=\sum_{j \in J} \alpha_{j}\langle 0| \prod_{k \in K_{j}} C\left(\lambda_{k}\right)$, with some computable coefficients $\alpha_{j}$ and sets $J$ and $K_{j}$ depending on $\mathcal{O}$ and $\left\langle\psi_{g}\right|$.

4 - Compute the correlation function as (multiple) sums of scalar products of states one of them being the ground state, $\left\langle\psi_{g}|\mathcal{O}| \psi_{g}\right\rangle=$ $=\sum_{j \in J} \alpha_{j}\left\langle 0\left|\prod_{k \in K_{j}} C\left(\lambda_{j}\right) \prod_{i \in I} B\left(\lambda_{i}\right)\right| 0\right\rangle$. Such scalar products can be computed using for example a new basis of the space of states induced by the so-called factorizing $F$-matrices (see [10]) and in which the operators $B$ and $C$ simplify drastically, hence allowing for a direct computation (for the $X X Z$ model it is given as a ratio of two determinants [7]).

5 - Take the thermodynamic limit (the limit of infinite lattice) of the above results where multiple sums leads to multiple integral representations of the correlation functions.

The above steps 1, 2, 3 can be achieved for a very general class of lattice integrable models. Steps 4 and 5 need more developments at the moment for the general models. However, $F$ basis has been determined for higher spin Heisenberg models in [11], using the functional Bethe ansatz technique of Sklyanin [38], and other models have been considered very recently in [39-41]. This gives some hope for a general solution of step 4 .

In the following sections, I will outline the application of this general method to the representative example of the $X X Z$ Heisenberg spin- $\frac{1}{2}$ in a magnetic field. Multiple integral represenations of its correlation functions have been obtained in [9]. This approach gives for this model a proof of the zero magnetic field expressions given by Jimbo, Miwa and their collaborators [6]. 


\section{The $\mathrm{XXZ}$ spin- $-\frac{1}{2}$ Heisenberg chain}

The Hamiltonian of the XXZ spin- $\frac{1}{2}$ Heisenberg chain of finite length $M[37,42]$ is given by,

$$
\begin{aligned}
& H_{\mathrm{XXZ}}=\sum_{m=1}^{M}\left\{\sigma_{m}^{x} \sigma_{m+1}^{x}+\sigma_{m}^{y} \sigma_{m+1}^{y}+\right. \\
& \left.+\Delta\left(\sigma_{m}^{z} \sigma_{m+1}^{z}-1\right)\right\},
\end{aligned}
$$

We impose periodic boundary conditions and $\sigma_{m}^{a}$, $a=x, y, z$, are the Pauli spin operators acting in the local quantum spin- $\frac{1}{2}$ space $\mathcal{H}_{m}$ at site $m$. The anisotropy parameter $\Delta$ defines the physical nature of the model: when $\Delta \leqslant-1$, the ground state of the Hamiltonian is ferromagnetic, whereas its magnetization is equal to zero when $\Delta>-1$. We shall focus our attention on this last domain, which itself decomposes into a massive regime (for $\Delta>1$ ), and a gapless regime (for $-1<\Delta<1$ ) in the thermodynamic limit $(M \rightarrow \infty)$.

The $R$-matrix of the $\mathrm{XXZ}$ model is,

$$
R(\lambda, \mu)=\left(\begin{array}{cccc}
1 & 0 & 0 & 0 \\
0 & b(\lambda, \mu) & c(\lambda, \mu) & 0 \\
0 & c(\lambda, \mu) & b(\lambda, \mu) & 0 \\
0 & 0 & 0 & 1
\end{array}\right)
$$

where the functions $b(\lambda, \mu)$ and $c(\lambda, \mu)$ are defined as,

$$
\begin{aligned}
& b(\lambda, \mu)=\frac{\sinh (\lambda-\mu)}{\sinh (\lambda-\mu+\eta)}, \\
& c(\lambda, \mu)=\frac{\sinh \eta}{\sinh (\lambda-\mu+\eta)} .
\end{aligned}
$$

The parameter $\eta$ is here related to the anisotropy parameter $\Delta$ of the Hamiltonian by,

$$
\Delta=\frac{1}{2}\left(q+q^{-1}\right), \quad \text { with } q=e^{\eta} .
$$

The $R$-matrix is a linear operator in the tensor product of two two-dimensional linear spaces $V_{1} \otimes V_{2}$, where each $V_{i}$ is isomorphic to $\mathbb{C}^{2}$, and depends generically on two spectral parameters $\lambda_{1}$ and $\lambda_{2}$ associated to these two vector spaces. It is denoted by $R_{12}\left(\lambda_{1}, \lambda_{2}\right)$. Such an $R$-matrix satisfies the Yang-Baxter equation (2.4). Moreover, it is unitary, except for a finite number of values of the spectral parameter, namely if $b\left(\lambda_{1}, \lambda_{2}\right) \neq \pm c\left(\lambda_{1}, \lambda_{2}\right)$ we have,

$$
R_{12}\left(\lambda_{1}-\lambda_{2}\right) R_{21}\left(\lambda_{2}-\lambda_{1}\right)=\mathbf{1},
$$

and reduces to the permutation operator for the particular value (zero) of the spectral parameter, $R_{12}(0)=P_{12}$.

Identifying one of the two vector spaces of the $R$-matrix with the quantum space $\mathcal{H}_{m}$, one defines the quantum $L$-operator of the inhomogeneous chain at site $m$ as,

$$
L_{m}\left(\lambda, \xi_{m}\right)=R_{0 m}\left(\lambda-\xi_{m}\right),
$$

where $\xi_{m}$ is an arbitrary inhomogeneity parameter attached to the site $m$. Here $R_{0 m}$ acts in $V_{0} \otimes$ $\mathcal{H}_{m}$, where $V_{0}$ is an auxiliary space isomorphic to $\mathbb{C}^{2}$. The monodromy matrix is constructed as an ordered product of such $L$-operators:

$$
\begin{aligned}
T_{0}(\lambda) & \equiv T_{0,1 \ldots N}\left(\lambda ; \xi_{1}, \ldots, \xi_{N}\right) \\
& =R_{0 N}\left(\lambda-\xi_{N}\right) \ldots R_{01}\left(\lambda-\xi_{1}\right),
\end{aligned}
$$

and can be represented in the auxiliary space $V_{0}$ as a $2 \times 2$ matrix,

$$
T(\lambda)=\left(\begin{array}{ll}
A(\lambda) & B(\lambda) \\
C(\lambda) & D(\lambda)
\end{array}\right)
$$

whose matrix elements $A, B, C, D$ are linear operators on the quantum space of states of the chain $\mathcal{H}=\otimes_{n=1}^{N} \mathcal{H}_{n}$.

The transfer matrix $\mathcal{T}(\lambda)$ is defined as the trace $A(\lambda)+D(\lambda)$ of the monodromy matrix. Transfer matrices commute with each other for different values of the spectral parameter $\lambda$. They commute also with the Hamiltonian (3.11) in the homogeneous case where all $\xi_{m}$ are equal as the Hamiltonian can be reconstructed in terms of the transfer matrix by means of the following "trace identity":

$$
H_{\mathrm{XXz}}=\left.2 \sinh \eta \partial_{\lambda} \log \mathcal{T}(\lambda)\right|_{\lambda=\xi_{j}}+\text { const. }
$$

Common eigenstates of the transfer matrices (and thus of the Hamiltonian (3.1) in the 
homogeneous case) can be constructed by successive actions of operators $B(\lambda)$ on the reference state $|0\rangle$, which is the ferromagnetic state with all the spins up. More precisely, the state $B\left(\lambda_{1}\right) \ldots B\left(\lambda_{N}\right)|0\rangle$ is a common eigenstate of the transfer matrices if the set of spectral parameters $\left\{\lambda_{j}\right\}_{1 \leqslant j \leqslant N}$ is a solution of the Bethe equations,

$$
\frac{a\left(\lambda_{j}\right)}{d\left(\lambda_{j}\right)} \prod_{\substack{k=1 \\ k \neq j}}^{N} \frac{b\left(\lambda_{j}, \lambda_{k}\right)}{b\left(\lambda_{k}, \lambda_{j}\right)}=1, \quad 1 \leqslant j \leqslant N
$$

where $a(\lambda)=1$ and $d(\lambda)=\prod_{i=1}^{M} b\left(\lambda, \xi_{i}\right)$ are the eigenvalues of operators $A(\lambda)$ and $D(\lambda)$ respectively on the reference state $|0\rangle$. The corresponding eigenvalue for the transfer matrix $\mathcal{T}(\mu)$ is then,

$$
\begin{aligned}
\tau\left(\mu,\left\{\lambda_{j}\right\}\right)= & a(\mu) \prod_{j=1}^{n} b^{-1}\left(\lambda_{j}, \mu\right)+ \\
& +d(\mu) \prod_{j=1}^{n} b^{-1}\left(\mu, \lambda_{j}\right) .
\end{aligned}
$$

The Bethe equations can also be written in a logarithmic form $(1 \leqslant j \leqslant N)$ :

$$
M p_{0_{\mathrm{tot}}}\left(\lambda_{j}\right)+\sum_{k=1}^{N} \theta\left(\lambda_{j}-\lambda_{k}\right)=2 \pi n_{j},
$$

where $n_{j}$ are integers for $N$ odd and half integers for $N$ even. The bare momentum $p_{0_{\text {tot }}}(\lambda)$ and the scattering phase $\theta(\lambda)$ are defined as,

$$
\begin{aligned}
p_{0_{\text {tot }}}(\lambda) & =\frac{i}{M} \ln \frac{d(\lambda)}{a(\lambda)} \\
& =\frac{1}{M} \sum_{k=1}^{M} p_{0}\left(\lambda-\xi_{k}+\frac{\eta}{2}\right), \\
p_{0}(\lambda) & =i \ln \frac{\sinh \left(\lambda-\frac{\eta}{2}\right)}{\sinh \left(\lambda+\frac{\eta}{2}\right)}, \\
\theta(\lambda) & =i \ln \frac{\sinh (\eta+\lambda)}{\sinh (\eta-\lambda)} .
\end{aligned}
$$

In the thermodynamic limit $(M \rightarrow \infty)$, these Bethe equations for the ground state become an integral equation for the quasi-particle density $\rho$ in the rapidity representation (Lieb equation) [43,
44]:

$$
\begin{array}{r}
\rho_{\mathrm{tot}}(\alpha)+\int_{-\Lambda}^{\Lambda} K(\alpha-\beta) \rho_{\mathrm{tot}}(\beta) d \beta= \\
=\frac{p_{0_{\mathrm{tot}}}^{\prime}(\alpha)}{2 \pi},
\end{array}
$$

where the new real variables $\alpha$ are defined in terms of general spectral parameters $\lambda$ differently in the two domains:

$$
\begin{array}{ll}
\alpha=\lambda \quad \text { for } \quad-1<\Delta<1, \\
\alpha=i \lambda \quad \text { for } \quad \Delta>1 .
\end{array}
$$

The density $\rho$ is defined as the limit of the quantity $\frac{1}{M\left(\alpha_{j+1}-\alpha_{j}\right)}, K(\alpha)$ and $p_{0_{\text {tot }}}^{\prime}(\alpha)$ are the derivatives with respect to $\alpha$ of the functions $-\frac{\theta(\lambda(\alpha))}{2 \pi}$ and $p_{0_{\text {tot }}}(\lambda(\alpha))$ :

$$
\begin{aligned}
K(\alpha) & =\frac{\sin 2 \zeta}{2 \pi \sinh (\alpha+i \zeta) \sinh (\alpha-i \zeta)} \\
p_{0}^{\prime}(\alpha) & =\frac{\sin \zeta}{\sinh \left(\alpha+i \frac{\zeta}{2}\right) \sinh \left(\alpha-i \frac{\zeta}{2}\right)}
\end{aligned}
$$

for $-1<\Delta<1$, with $\zeta=i \eta$ and,

$$
\begin{aligned}
K(\alpha) & =\frac{\sinh 2 \zeta}{2 \pi \sin (\alpha+i \zeta) \sin (\alpha-i \zeta)} \\
p_{0}^{\prime}(\alpha) & =\frac{\sinh \zeta}{\sin \left(\alpha+i \frac{\zeta}{2}\right) \sin \left(\alpha-i \frac{\zeta}{2}\right)}
\end{aligned}
$$

for $\Delta>1$, with $\zeta=-\eta$, and where $\beta_{k}=\xi_{k}$ in the domain $-1<\Delta<1$, and $\beta_{k}=i \xi_{k}$ in the domain $\Delta>1$. The integration limit $\Lambda$ is equal to $\frac{\pi}{2}$ for $\Delta>1$, and to $+\infty$ for $-1<\Delta<1$.

The solution for the Lieb equation ( $\overline{3} . \overline{1} \overline{1})$ in the homogeneous model where all parameters $\xi_{k}$ are equal to $\eta / 2$, that is the density for the ground state of the Hamiltonian (3.1) in the thermodynamic limit, is given by the following function [44]:

$$
\begin{aligned}
& \rho(\alpha)=\frac{1}{2 \zeta \cosh \left(\frac{\pi \alpha}{\zeta}\right)} \text { for }-1<\Delta<1, \\
& \rho(\alpha)=\frac{1}{2 \pi} \sum_{n=-\infty}^{+\infty} \frac{e^{2 i n \alpha}}{\cosh (n \zeta)} \text { for } \Delta>1 .
\end{aligned}
$$


For technical convenience, we will also use in the following the solution of the inhomogeneous Lieb equation, that is the function,

$$
\rho_{\mathrm{tot}}(\alpha)=\frac{1}{M} \sum_{i=1}^{M} \rho\left(\alpha-\beta_{k}-i \frac{\zeta}{2}\right) .
$$

It will be also convenient to consider, without any loss of generality, that the inhomogeneity parameters are contained in the region $-\zeta<\operatorname{Im} \beta_{j}<0$.

Let us mention at last that the ground state of the XXZ model in the region $\Delta>1$ is degenerated in the thermodynamic limit $(M \rightarrow \infty)$, namely there are two states with the same energy (and characterized by the same density $(\overline{3} . \overline{1} \overline{3})$ ), which we will call the ground state $\left|\Psi_{1}\right\rangle$ and the quasi-ground state $\left|\Psi_{2}\right\rangle$ (on the finite lattice, these states possess different energy). In this domain, the correlation function at zero temperature is thus half of the trace on these two states, that is of the sum of the two corresponding matrix elements. In the domain $-1<\Delta<1$, the ground state is not degenerated.

\section{The quantum inverse scattering problem}

The solution of this problem was a dream during many years for most of us working in the domain of quantum integrable models. It was shown for the first time in [7] that the explicit solution of the quantum inverse scattering problem (namely the reconstruction of any local spin operator at any site of the chain in terms of the elements of the quantum monodromy matrix satisfying a Yang-Baxter algebra, and containing creation/annihilation operators of Bethe eigenstates of the Hamiltonian) can be solved for the $X X Z$ spin- $\frac{1}{2}$ model in a very simple and purely algebraic way.

The elementary nature of the answer in this very representative example among the models solvable by means of the algebraic Bethe ansatz method, was quite unexpected. The algebraic Bethe ansatz method, also called quantum inverse scattering method, appeared twenty years ago as a quantum analogue of the classical inverse scattering problem approach to non-linear wave equations having soliton solutions, in order to solve quantum integrable models in two dimensions. The essential tools of this method are the quantum monodromy matrix satisfying quadratic commutation relations (Yang-Baxter algebra) which structures constants are given by the corresponding $R$-matrix solving the YangBaxter (cubic) equation $[1,35]$. It has been designed to diagonalize the corresponding Hamiltonians simultaneously with their associated commuting family of integrals of motion $[12,45]$. This method is in fact the quantum analogue of the $d i$ rect part of the classical inverse scattering problem method in its Hamiltonian formulation [46], in which the Lax matrix is used to construct the monodromy matrix containing the action-angle variables which linearize the dynamics. However, the inverse scattering problem part of the classical theory, namely, the reconstruction of the local classical field variables contained in the Lax matrix in terms of the elements of the monodromy matrix (and hence in terms of the action-angle variables) using the Gel'fand-Levitan-Marchenko equations [47-49], being already a quite difficult problem to solve, it was not at all obvious to find a direct way to extend it to the quantum situation, although the motivations (form factors and correlation functions) were clear from the very beginning [50-52].

The very remarkable feature of the solution to this problem given in [7] was not only that the quantum inverse scattering problem can be solved explicitely, but also that both its resulting expressions (reconstruction of the local spin operators at any site of the chain in terms of a simple product of the quantum monodromy matrix elements) and their proofs are very elementary. In turn, it essentially relies on the fact that the quantum $R$-matrix $R(\lambda, \mu)$ solving the YangBaxter equation reduces to the permutation operator when the two spectral parameters $\lambda$ and $\mu$ are equal.

This fact being almost a consequence of the Yang-Baxter equation itself, and hence satisfied for very generic cases, it immediatly suggested that the quantum inverse scattering problem can indeed be solved for almost all known quantum 
integrable (lattice) models. This is achieved for a large class of integrable lattice models in [13]. It is to be compared to the tentatives of solving the corresponding problem directly for the continuum quantum integrable field theories such as the Sine-Gordon model, using a quantization of the Gel'fand-Levitan-Marchenko equations, which appeared to be an extremely difficult problem to handle, due in particular to the presence of boundstates [4].

Let us now describe the solution for the $X X Z$ spin- $\frac{1}{2}$ model $[7,13]$. The result and its proof can be easily extended to very general lattice models, including the so-called fundamental lattice models, but also the models with impurities and the fusion models (see [13]).

THEOREM 1. Local spin operators at a given site $i$ of the inhomogeneous XXZ Heisenberg chain are given by

$$
\begin{aligned}
\sigma_{i}^{-}=\prod_{\alpha=1}^{i-1}(A+D)\left(\xi_{\alpha}\right) \cdot B\left(\xi_{i}\right) \cdot \\
\cdot \prod_{\alpha=1}^{i}(A+D)^{-1}\left(\xi_{\alpha}\right) \\
\sigma_{i}^{+}=\prod_{\alpha=1}^{i-1}(A+D)\left(\xi_{\alpha}\right) \cdot C\left(\xi_{i}\right) \cdot \\
\cdot \prod_{\alpha=1}^{i}(A+D)^{-1}\left(\xi_{\alpha}\right) \\
\sigma_{i}^{z}=\prod_{\alpha=1}^{i-1}(A+D)\left(\xi_{\alpha}\right) \cdot(A-D)\left(\xi_{i}\right) . \\
\cdot \prod_{\alpha=1}^{i}(A+D)^{-1}\left(\xi_{\alpha}\right) .
\end{aligned}
$$

Note that the case of homogeneous models where all $\xi$ are equal is also included in this result by just taking the wanted specific equal values for the $\xi_{j}$.

\section{From the inverse scattering prob- lem to correlation functions}

Using the above solution to the quantum inverse scattering problem for local spins, we can compute the correlation functions. For this we need three more ingredients, which are the computation of generic actions of local operators on the ground state using Yang-Baxter commutation relations, the scalar product of states, one of them being a Bethe state, and finally a simple procedure to take the thermodynamic limit. These tools are given in the next subsections.

\subsection{Action of operators $A, B, C, D$ on a general state}

We want now to express the action of any local operator on a state constructed by action of $C$ operators on the reference state. This is given in turn from the solution of the quantum inverse scattering problem by the successive action of some product of $A, B, C, D$ operators. Action of $A, B, C, D$ on such a state are well known (see for example [5]), but we recasted them [9] in a more convenient form for our purpose.

The action of the operators $A(\lambda)$ and $D(\lambda)$ on the states constructed by successive actions of operators $C(\lambda)$ can be written in the following form:

$$
\begin{aligned}
& \langle 0| \prod_{k=1}^{N} C\left(\lambda_{k}\right) A\left(\lambda_{N+1}\right)=\sum_{a^{\prime}=1}^{N+1} a\left(\lambda_{a^{\prime}}\right) .
\end{aligned}
$$

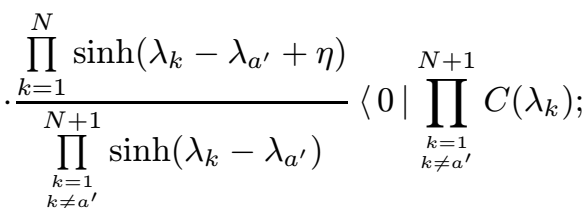

$$
\begin{aligned}
& \langle 0| \prod_{k=1}^{N} C\left(\lambda_{k}\right) D\left(\lambda_{N+1}\right)=\sum_{a=1}^{N+1} d\left(\lambda_{a}\right) . \\
& \frac{\prod_{\substack{k=1 \\
k=1 \\
k \neq a}}^{N+1} \sinh \left(\lambda_{a}-\lambda_{k}+\eta\right)}{N+1}\langle 0| \prod_{\substack{k=1 \\
k \neq a}}^{N+1} C\left(\lambda_{k}\right) .
\end{aligned}
$$

The action of the operator $B(\lambda)$ is more complicated and involved a double sum (see [9]), but in the case which is interesting for the computation of the correlation functions when $\lambda_{N+1}=\xi_{k}$ and hence $d\left(\lambda_{N+1}\right)=0$, we obtain a more simple 
result:

$$
\begin{gathered}
\langle 0| \prod_{k=1}^{N} C\left(\lambda_{k}\right) B\left(\lambda_{N+1}\right)=\sum_{a=1}^{N} d\left(\lambda_{a}\right) \cdot \\
\frac{\prod_{k=1}^{N} \sinh \left(\lambda_{a}-\lambda_{k}+\eta\right)}{\prod_{\substack{k=1 \\
k \neq a}}^{N+1} \sinh \left(\lambda_{a}-\lambda_{k}\right)} \sum_{\substack{a^{\prime}=1 \\
a^{\prime} \neq a}}^{N+1} a\left(\lambda_{a^{\prime}}\right) \cdot \\
\frac{\prod_{\substack{j=1 \\
j \neq a}}^{N} \sinh \left(\lambda_{j}-\lambda_{a^{\prime}}+\eta\right)}{\prod_{\substack{j=1 \\
j \neq a, a^{\prime}}}^{N+1} \sinh \left(\lambda_{j}-\lambda_{a^{\prime}}\right)}\langle 0| \prod_{\substack{k=1 \\
k \neq a, a^{\prime}}}^{N+1} C\left(\lambda_{k}\right) .
\end{gathered}
$$

It should be mentioned that the action of $B$ is similar to the successive action of $D$ and $A$. Using these formulae, one can reduce any expressions for general correlation functions to (multiple) sums of scalar products of a Bethe state with an arbitrary state constructed by successive actions of $B$ operators on the reference state.

\subsection{Scalar products}

The next step of the computation is to find an explicit and convenient expression for scalar products of two states one of them being a Bethe eigenstate. Usual Bethe ansatz techniques, based only on the use of commutation relations, generally generate huge sums which are difficult to sum up. In [7], a direct computation in a new basis ( $F$-basis) [10] has been performed and leads to an explicit expression for such scalar products as a determinant of usual functions of the model:

Theorem 2. Let $\left\{\lambda_{1}, \ldots, \lambda_{N}\right\}$ be a solution of the Bethe equations (12.6) and $\left\{\mu_{1}, \ldots, \mu_{N}\right\}$ be an arbitrary set of parameters. Then the scalar product,

$$
\begin{gathered}
S_{N}\left(\left\{\mu_{j}\right\},\left\{\lambda_{k}\right\}\right)=\langle 0| \prod_{j=1}^{N} C\left(\mu_{j}\right) . \\
\cdot \prod_{k=1}^{N} B\left(\lambda_{k}\right)|0\rangle
\end{gathered}
$$

can be represented as a ratio of two determinants,

$$
\begin{aligned}
S_{N}\left(\left\{\mu_{j}\right\},\left\{\lambda_{k}\right\}\right) & =S_{N}\left(\left\{\lambda_{k}\right\},\left\{\mu_{j}\right\}\right) \\
& =\frac{\operatorname{det} T\left(\left\{\mu_{j}\right\},\left\{\lambda_{k}\right\}\right)}{\operatorname{det} V\left(\left\{\mu_{j}\right\},\left\{\lambda_{k}\right\}\right)}
\end{aligned}
$$

of the following $N \times N$ matrices $T$ and $V$ :

$$
\begin{aligned}
& T_{a b}=\partial_{\lambda_{a}} \tau\left(\mu_{b},\left\{\lambda_{k}\right\}\right), \\
& V_{a b}=\frac{1}{\sinh \left(\mu_{b}-\lambda_{a}\right)}, \quad 1 \leqslant a, b \leqslant N,
\end{aligned}
$$

where $\tau\left(\mu_{b},\left\{\lambda_{k}\right\}\right)$ is the eigenvalue of the transfer matrix $\mathcal{T}\left(\mu_{b}\right)$ corresponding to the Bethe state $\prod_{k=1}^{N} B\left(\lambda_{k}\right)|0\rangle$ given by (13.9).

This result is equivalent to the scalar product formula obtained in [53].

When particularizing this formula in the case when the two states are equal, one obtains the Gaudin formula for the norm of a Bethe state:

$$
\begin{gathered}
\left\langle 0\left|\prod_{j=1}^{N} C\left(\lambda_{j}\right) \prod_{k=1}^{N} B\left(\lambda_{k}\right)\right| 0\right\rangle=\sinh ^{N} \eta . \\
\cdot \prod_{\alpha \neq \beta} \frac{\sinh \left(\lambda_{\alpha}-\lambda_{\beta}+\eta\right)}{\sinh \left(\lambda_{\alpha}-\lambda_{\beta}\right)} \operatorname{det} \Phi^{\prime}\left(\left\{\lambda_{a}\right\}\right),
\end{gathered}
$$

where $\Phi^{\prime}$ is a $N \times N$ matrix the elements of which are given by:

$$
\Phi_{a b}^{\prime}=-\partial_{\lambda_{b}} \ln \left(\frac{a\left(\lambda_{a}\right)}{d\left(\lambda_{a}\right)} \prod_{\substack{k=1 \\ k \neq a}}^{N} \frac{b\left(\lambda_{a}, \lambda_{k}\right)}{b\left(\lambda_{k}, \lambda_{a}\right)}\right) .
$$

By means of this expression for the scalar product, general correlation functions for the finite chain can now be expressed as sums of determinants.

\subsection{Thermodynamic limit}

The last step of our method, to obtain the general correlation functions in the infinite volume limit, is to take the thermodynamic limit of the expressions obtained for the finite chain. In the thermodynamic limit $M \rightarrow \infty$, the Bethe equations for the ground state become the integral 
Lieb equation (13.11) for the density. In a more general way, for any $\mathcal{C}^{\infty}$ function $f(\pi$-periodic in the domain $\Delta>1$ ), sums over all the values of $f$ at the point $\alpha_{j}, 1 \leqslant j \leqslant N$, parameterizing the ground state, can be replaced in the thermodynamic limit by an integral involving the density $\rho$ solution of the Lieb equation [8]:

$$
\begin{array}{r}
\frac{1}{M} \sum_{j=1}^{N} f\left(\alpha_{j}\right)=\int_{-\Lambda}^{\Lambda} f(\alpha) \rho_{\text {tot }}(\alpha) d \alpha+ \\
+O\left(M^{-\infty}\right) .
\end{array}
$$

Thus, sums over determinants will become multiple integrals.

These properties enabled us in [8] to obtain the expression of the Gaudin matrix elements $(15.8)$ in the thermodynamic limit:

$$
\begin{gathered}
\Phi_{a b}^{\prime}(\alpha)=-2 i \pi M\left\{\delta_{a b} \rho_{\mathrm{tot}}\left(\alpha_{a}\right)+\right. \\
\left.+\frac{1}{M} K\left(\alpha_{a}-\alpha_{b}\right)\right\}+O\left(M^{-\infty}\right) \\
\text { for }-1<\Delta<1, \\
\Phi_{a b}^{\prime}(\alpha)=2 \pi M\left\{\delta_{a b} \rho_{\mathrm{tot}}\left(\alpha_{a}\right)+\right. \\
\left.+\frac{1}{M} K\left(\alpha_{a}-\alpha_{b}\right)\right\}+O\left(M^{-\infty}\right) \\
\text { for } \Delta>1 .
\end{gathered}
$$

These expressions will be useful in the following to compute the determinants which appear in the formulae for the correlation functions in the thermodynamic limit. Finally, we will obtain correlation functions as multiple integrals of usual functions of the model.

\section{Correlation functions}

The general formula for the correlation functions of the XXZ Heisenberg spin- $\frac{1}{2}$ chain in a magnetic field were given in [9]. Here, I will simply describe the structure of such a formula in terms of multiple integrals for the generic building blocks of correlation functions defined by,

$$
F_{m}\left(\left\{\epsilon_{j}, \epsilon_{j}^{\prime}\right\}\right)=\frac{\left\langle\psi_{g}\left|\prod_{j=1}^{m} E_{j}^{\epsilon_{j}^{\prime}, \epsilon_{j}}\right| \psi_{g}\right\rangle}{\left\langle\psi_{g} \mid \psi_{g}\right\rangle} .
$$

where, $E_{m}^{\epsilon_{m}^{\prime}, \epsilon_{m}}$ are the elementary operators acting on $\mathcal{H}_{m}$ at site $m$ as the $2 \times 2$ matrices $E_{l k}^{\epsilon^{\prime}, \epsilon}=$ $\delta_{l, \epsilon^{\prime}} \delta_{k, \epsilon}$, and $\prod_{j=1}^{m} E_{j}^{\epsilon_{j}^{\prime}, \epsilon_{j}}$ is any product of such elementary operators from site one to $m$. Any $n$-point correlation function can be obtained as sums of these building blocks. To calculate this product we use the solution of the quantum inverse scattering problem, representing the local elementary matrices in terms of the corresponding monodromy matrix elements:

$$
\begin{aligned}
E_{j}^{\epsilon_{j}^{\prime}, \epsilon_{j}} & =\prod_{k=1}^{j-1}\left(A\left(\xi_{k}\right)+D\left(\xi_{k}\right)\right) . \\
& \cdot T_{\epsilon_{j}, \epsilon_{j}^{\prime}}\left(\xi_{j}\right) \prod_{k=1}^{j}\left(A\left(\xi_{k}\right)+D\left(\xi_{k}\right)\right)^{-1} .
\end{aligned}
$$

Thus we reduce the problem to the computation of the ground state mean value of an arbitrary ordered product of the monodromy matrix elements,

$$
\begin{array}{r}
F_{m}\left(\left\{\epsilon_{j}, \epsilon_{j}^{\prime}\right\}\right)=\phi_{m}(\{\lambda\}) \\
\cdot \frac{\left\langle\psi_{g}\left|T_{\epsilon_{1}, \epsilon_{1}^{\prime}}\left(\xi_{1}\right) \ldots T_{\epsilon_{m}, \epsilon_{m}^{\prime}}\left(\xi_{m}\right)\right| \psi_{g}\right\rangle}{\left\langle\psi_{g} \mid \psi_{g}\right\rangle}
\end{array}
$$

where $\phi_{m}(\{\lambda\})$ is the ground state eigenvalue of the corresponding product of the transfer matrices:

$$
\phi_{m}(\{\lambda\})=\prod_{j=1}^{m} \prod_{a=1}^{N} \frac{\sinh \left(\lambda_{a}-\xi_{j}\right)}{\sinh \left(\lambda_{a}-\xi_{j}+\eta\right)} .
$$

Now to calculate these mean values we use the commutation relations of the monodromy matrix elements. An arbitrary product of the monodromy matrix elements can be treated in a rather general way. One should consider the two following sets of indices:

$$
\begin{aligned}
& \alpha^{+}=\left\{j: 1 \leqslant j \leqslant m, \epsilon_{j}=1\right\}, \\
& \max _{j \in \alpha^{+}}(j) \equiv j_{\max }^{\prime}, \min _{j \in \alpha^{+}}(j) \equiv j_{\min }^{\prime}, \\
& \alpha^{-}=\left\{j: 1 \leqslant j \leqslant m, \epsilon_{j}^{\prime}=2\right\} \\
& \max _{j \in \alpha^{-}}(j) \equiv j_{\max }, \min _{j \in \alpha^{-}}(j) \equiv j_{\min } .
\end{aligned}
$$

We also note $\operatorname{card}\left(\alpha^{+}\right)=s^{\prime}$ and $\operatorname{card}\left(\alpha^{-}\right)=s$. It should be mentioned that in a general case the intersection of these two sets is not empty and corresponds to the operators $B\left(\xi_{j}\right)$.

Consider now the action of an arbitrary product of monodromy matrix elements on a state 
constructed by the action of the operators $C(\lambda)$,

$$
\begin{array}{r}
\langle 0| \prod_{k=1}^{N} C\left(\lambda_{k}\right) T_{\epsilon_{1}, \epsilon_{1}^{\prime}}\left(\lambda_{N+1}\right) \ldots \\
\ldots T_{\epsilon_{m}, \epsilon_{m}^{\prime}}\left(\lambda_{N+m}\right),
\end{array}
$$

applying one by one the formulae $(\overline{5} \cdot \overline{1})-(\overline{5} \cdot \overline{3})$. For all the indices $j$ from the sets $\alpha^{+}$and $\alpha^{-}$one obtains a summation on the corresponding indices $a_{j}^{\prime}$ (for $j \in \alpha^{+}$, corresponding to the action of the operators $A(\lambda)$ or $B(\lambda)$ ) or $a_{j}$ (for $j \in \alpha^{-}$, corresponding to the action of the operators $D(\lambda)$ or $B(\lambda)$ ). As the product of the monodromy matrix elements is ordered these summations are also ordered and the corresponding indices should be taken from the following subsets of integers $b$, $1 \leqslant b \leqslant N+m$

$$
\begin{aligned}
\mathbf{A}_{j} & =\left\{b: b \neq a_{k}, a_{k}^{\prime}, k<j\right\}, \\
\mathbf{A}_{j}^{\prime} & =\left\{b: b \neq a_{k}^{\prime}, k<j, b \neq a_{k}, k \leqslant j\right\} .
\end{aligned}
$$

Thus the action of a product of the monodromy matrix elements can be written as the following sum:

$$
\begin{gathered}
\langle 0| \prod_{k=1}^{N} C\left(\lambda_{k}\right) T_{\epsilon_{1}, \epsilon_{1}^{\prime}}\left(\lambda_{N+1}\right) \ldots \\
\ldots T_{\epsilon_{m}, \epsilon_{m}^{\prime}}\left(\lambda_{N+m}\right)=\sum_{\left\{a_{j}, a_{j}^{\prime}\right\}} G_{\left\{a_{j}, a_{j}^{\prime}\right\}}\left(\lambda_{i}\right) \times \\
\times\langle 0| \prod_{b \in \mathbf{A}_{m+1}} C\left(\lambda_{b}\right)
\end{gathered}
$$

The summation is taken over the indices $a_{j}$ for $j \in \alpha^{-}$and $a_{j}^{\prime}$ for $j \in \alpha^{+}$such that:

$$
\begin{aligned}
& 1 \leqslant a_{j} \leqslant N+j, a_{j} \in \mathbf{A}_{j}, \\
& 1 \leqslant a_{j}^{\prime} \leqslant N+j, a_{j}^{\prime} \in \mathbf{A}_{j}^{\prime} .
\end{aligned}
$$

The functions $G_{\left\{a_{j}, a_{j}^{\prime}\right\}}\left(\lambda_{1}, \ldots \lambda_{N+m}\right)$ can be easily obtained from the formulae $(5.1)(1-3)$ taking into acount that $\lambda_{a}=\xi_{N-a}$ for $a>N$ :

$$
\begin{array}{r}
G_{\left\{a_{j}, a_{j}^{\prime}\right\}}\left(\lambda_{1}, \ldots, \lambda_{N+m}\right)= \\
\prod_{j \in \alpha^{-}} d\left(\lambda_{a_{j}}\right) \frac{\prod_{\substack{b=1 \\
b \in \mathbf{A}_{j}}}^{N+j-1} \sinh \left(\lambda_{a_{j}}-\lambda_{b}+\eta\right)}{\prod_{\substack{b=1 \\
b \in \mathbf{A}_{j}^{\prime} \\
N+j}}^{N i n h}\left(\lambda_{a_{j}}-\lambda_{b}\right)} \times \\
\times \prod_{j \in \alpha^{+}} a\left(\lambda_{a_{j}^{\prime}}\right) \frac{\prod_{\substack{b=1 \\
b \in \mathbf{A}_{j}^{\prime}}}^{\prod_{j}+j} \sinh \left(\lambda_{b}-\lambda_{a_{j}^{\prime}}+\eta\right)}{\prod_{b=1}^{N+j} \sinh \left(\lambda_{b}-\lambda_{a_{j}^{\prime}}\right)} .
\end{array}
$$

Now to calculate the normalized mean value (16.2) we apply the representation for the scalar product (15.5) and the Gaudin formula (15.7). Going to the thermodynamic limit, multiple sums become multiple integrals, and finally, we obtained the expression of these correlation functions in the homogeneous limit where all $\xi$ are equal. It is given as a $m$ fold integral over contours $C_{j}^{h}$ which are depending on the value of $j$, on the regime considered and also on the value of the magnetic field. The general answer can be written as [9],

$$
\begin{aligned}
& F_{m}\left(h,\left\{\epsilon_{j}, \epsilon_{j}^{\prime}\right\}\right)=\prod_{j=1}^{m} \int_{C_{j}^{h}} d \lambda_{j} . \\
& \cdot \Omega_{m}\left(\left\{\lambda_{j}\right\},\left\{\epsilon_{j}, \epsilon_{j}^{\prime}\right\}\right) \operatorname{det} S_{h}(\{\lambda\}) .
\end{aligned}
$$

where $\Omega_{m}\left(\left\{\lambda_{j}\right\},\left\{\epsilon_{j}, \epsilon_{j}^{\prime}\right\}\right)$ is a purely algebraic quantity, not depending on the regime or on the magnetic field, while $S_{h}(\{\lambda\})$ is a matrix depending on the density function $\rho_{h}(\lambda)$ describing the ground state, solution of the Lieb equation, and hence which depends both on the regime and on the value of the magnetic field $h$.

\section{Conclusions}

We have described a general approach to compute correlation functions of lattice quantum integrable models, with the XXZ Heisenberg spin$\frac{1}{2}$ chain in a magnetic field as a typical example. The main ingredients are the solution of the quantum inverse scattering problem $[7,13]$ and the scalar product formula $[7,53]$. 
Our method should now be extended to temperature and time dependent correlation functions. Another very important problem concerns the long distance asymptotics and the scaling limit (the limit in which the lattice spacing goes to zero, while the number of sites $N$ goes to infinity, their product being kept fixed). This would open the possibility to consider also the quantum integrable field theory models. Another route towards this goal would be to solve the quantum inverse problem directly for the lattice versions of quantum integrable field theory models, along the lines described in [13] for higher spin Heisenberg chains. In this context, we will have to deal with quantum Lax operators having infinite dimensional representation auxiliary space. I expect the resulting reconstruction expressions for the local quantum fields, at least for the most simple cases, to be given by formulas very similar to the infinite spin limit of the result for spin- $s$ Heisenberg chains [13].

Acknowledgements. I would like to thank here my collaborators on this difficult subject, J. Sanchez de Santos, N. Kitanine, and V. Terras. I also would like to express a particular thought for our friend and collaborator A. Izergin who passed away last year.

\section{References}

[1] R.J. Baxter, Exactly solved models in statistical mechanics (Academic Press, London - New York, 1982).

[2] L.D. Faddeev, Les Houches 1982, Recent advances in field theory and statistical mechanics, edited by J.B. Zuber and R. Stora, pp. 561-608, Elsevier Science Publ., 1984.

[3] M. Gaudin, La fonction d'onde de Bethe (Masson, 1983)

[4] F.A. Smirnov, Form factors in completely integrable models of quantum field theory (World Scientific, Singapore, 1992).

[5] V.E. Korepin, N.M. Bogoliubov and A.G. Izergin, Quantum inverse scattering method and correlation functions (Cambridge University Press, 1993).
[6] M. Jimbo and T. Miwa, Algebraic analysis of solvable lattice models (AMS, 1995).

[7] N. Kitanine, J.M. Maillet and V. Terras, Nucl. Phys. B 554 [FS] (1999) 647, math-ph/9807020.

[8] A.G. Izergin et al., Nucl. Phys. B 554 [FS] (1999) 679, solv-int/9812021.

[9] N. Kitanine, J.M. Maillet and V. Terras, Nucl. Phys. B 567 [FS] (2000) 554, math-ph/9907019.

[10] J.M. Maillet and J. Sanchez de Santos, Drinfel'd twists and algebraic Bethe Ansatz, in L. D. Faddeev's Seminar on Mathematical Physics, Advances in the Mathematical Sciences, vol. 201, M. Semenov-Tian-Shansky editor, AMS (2000). q-alg/9612012, 1996.

[11] V. Terras, Lett. Math. Phys. 48 (1999) 263, math-ph/9902009.

[12] L.D. Faddeev, E.K. Sklyanin and L.A. Takhtajan, Theor. Math. Phys. 40 (1980) 688, Translated from Teor. Mat. Fiz. 40, 1979.

[13] J.M. Maillet and V. Terras, Nucl. Phys. B 575 [FS] (2000) 627, hep-th/9911030.

[14] C.N. Yang, Phys. Rev. 85 (1952) 808.

[15] T.T. Wu et al., Phys. Rev. B13 (1976) 316.

[16] M. Jimbo et al., Physica 1 D (1980) 80.

[17] A.A. Belavin, A.M. Polyakov and A.B. Zamolodchikov, Nucl. Phys. B 241 (1984) 333.

[18] V.G. Knizhnik and A.B. Zamolodchikov, Nucl. Phys. B 247 (1984) 83.

[19] A.B. Zamolodchikov and A.B. Zamolodchikov, Ann. Phys. 120 (1979) 253.

[20] M. Karowski and P. Weisz, Nucl. Phys. B 139 (1978) 445.

[21] M. Karowski and H.J. Thun, Nucl. Phys. B 190 (1981) 61.

[22] I.B. Frenkel and N.Y. Reshetikhin, Commun. Math. Phys. 146 (1992) 1.

[23] E. Date, M. Jimbo and M. Okado, Commun. Math. Phys. 155 (1993) 47.

[24] M. Jimbo et al., J. Phys. A27 (1994) 3267, hepth/9309118.

[25] F.A. Smirnov, RIMS preprint 860 (1992).

[26] F.A. Smirnov, Commun. Math. Phys. 155 (1993) 459, hep-th/9210052.

[27] F.A. Smirnov, RIMS preprint 935 (1993).

[28] A. Varchenko, Comm. Math. Phys. 162 (1994) 499. 
[29] R.J. Baxter, J. Stat. Phys. 15 (1976) 485.

[30] R.J. Baxter, J. Stat. Phys. 17 (1977) 1.

[31] H.B. Thacker, Physica D18 (1986) 348.

[32] M. Jimbo et al., Phys. Lett. A 168 (1992) 256.

[33] M. Jimbo, T. Miwa and A. Nakayashiki, J. Phys. A26 (1993) 2199, hep-th/9211066.

[34] A.R. Its and N.A. Slavnov, On the RiemannHilbert approach to the asymptotic analysis of the correlation functions of the quantum nonlinear Schrödinger equation. Non-free fermionic case., Preprint MI-98-76, math-ph/9811009, 1998.

[35] C.N. Yang, Phys. Rev. Lett. 19 (1967) 1312.

[36] R.J. Baxter, Ann. Phys. 70 (1972) 193.

[37] H. Bethe, Zeitschrift fr Physik 71 (1931) 205.

[38] E.K. Sklyanin, Functional Bethe Ansatz, Integrable and Superintegrable Systems, edited by B. Kupershmidt, pp. 8-33, World Scientific, Singapore, 1990.

[39] T.-D. Albert, H. Boos, R. Flume, K. Ruhlig Resolution of the Nested Hierarchy for Rational sl(n) Models J. Phys. A33 (2000) 4963, nlin.SI/0002027.

[40] T.-D. Albert, H. Boos, R. Flume, R.H. Poghossian, K. Ruhlig The Drinfel'd twisted XYZ model nlin.SI/0005023.

[41] T.-D. Albert, K. Ruhlig Polarization-free generators for the Belavin model nlin.SI/0007020.

[42] W. Heisenberg, Zeitschrift für Physik 49 (1928) 619.

[43] E.H. Lieb and W. Liniger, Phys. Rev. 130 (1963) 1605.

[44] C.N. Yang and C.P. Yang, Phys. Rev. 150 (1966) 327.

[45] L.A. Takhtajan and L.D. Faddeev, Russ. Math. Surveys 34 (1979) 11.

[46] V.E. Zakharov and L.D. Faddeev, Funct. Anal. Appl. 5 (1971) 280.

[47] I.M. Gel'fand and B.M. Levitan, Am. Math. Soc. Transl. Ser. 21 (1955) 253.

[48] V.A. Marchenko, Dokl. Akad. Nauk SSSR 104 (1955) 695, In russian.

[49] M.J. Ablowitz et al., Phys. Rev. Lett. 30 (1973) 1262 .

[50] D.B. Creamer, H.B. Thacker and D. Wilkinson, Phys. Rev. D 21 (1980) 1523.
[51] D.B. Creamer, H.B. Thacker and D. Wilkinson, Phys. Lett. 92 B (1980) 144.

[52] D.B. Creamer, H.B. Thacker and D. Wilkinson, Phys. Rev. D 23 (1981) 3081.

[53] N.A. Slavnov, Theor. Math. Phys. 79 (1989) 502 .

[54] M. Jimbo and T. Miwa, Journ. Phys. A: Math. Gen. 29 (1996) 2923. 